\title{
Comunidades de crustáceos de las lagunas de Tordera (Barcelona) en relación con sus características ambientales
}

\author{
Francesc Sabater
}

Departamento de Ecología, Facultad de Biología, Universidad de Barcelona.

\begin{abstract}
SUMMARY
CRUSTACEAN COMMUNITIES IN TORDERA SMALL WATER BODIES (BARCELONA) IN CONNECTION WITH ENVIRONMENTAL CHARACTERISTICS

Crustacean communities from several temporal ponds were studied biweekly from September 1980 to October 1981. Four types of ponds were distinguished based on morphological, physical and chemical characteristics. The crustacean communities are related to these characteristics. Seasonality is the most important factor to be considered because it accounts for the evolutionary degree that the communities reach.
\end{abstract}

\section{INTRODUCCION}

Los estudios limnológicos sobre los sistemas de lagunas de carácter temporal y los ambientes acuáticos de extensión reducida, hari sido pocos hasta el momento, en comparación con los efectuados en lagos u otros tipos de sistemas acuáticos de mayor superficie.

Este tipo de ambientes se caracterizan por presentar una gran variabilidad en el tiempo $y$ en el espacio. Mientras que la variabilidad en el tiempo se manifiesta por la inestabilidad del sistema, la variabilidad en el espacio se refleja en una gran variedad de microclimas que en las regiones templadas, han sido definidos por la población vegetal que los coloniza (DUSSART, 1966). Por otra parte, este tipo de ambientes presenta una cierta heterogeneidad de biotopos (TETART 1974).

Es caracteristico de este tipo de sistemas fluctuantes de poca profundidad el poseer un intenso "metabolismo" (PROSZYNSKA 1962a), ya que existe una rápida mineralización de sustancias orgánicas y una inmediata asimilación por parte de las plantas higrófilas. Por su poca profundidad, el agua más profunda se calienta rápidamente, originando corrientes de convección que mezclan el agua. Se consideran por tanto sistemas polimícticos (DUSSART, 1966). Fruto de este intenso metabolismo es la elevada tasa de multiplicación y el rápido desarrollo que presentan la fauna y la flora que coloniza este tipo de ambientes.

Sean cuales sean las características de los sistemas de lagunas de carácter temporal y su 
localización geográfica, tienen en común una serie de peculiaridades físicas y biológicas de gran interés, que-han atraído la atención de naturalistas y fisiólogos interesados en el estudio de los crustáceos.

\section{DESCRIPCION DE LA ZONA}

El sistema de lagunas de "Ca l'Estany de Tordera" se encuentra situado en la parte baja del curso del río "la Tordera" a $5 \mathrm{Km}$. aproximadamente de su desembocadura y a unos $2 \mathrm{Km}$. de la población de Tordera ( $\mathrm{Co}$ marca del Maresme, Barcelona) (fig. 1).

Bajo el punto de vista hidrológico, esta zona se encuentra dentro tei area de iniluencia del nivel freático del río el régimen del cual es exclusivamente pluvial ya que no existen acumulaciones importantes de nieve en su cuenca y por ello presenta un caudal medio mensual con muchas fluctuaciones, régimen propio de los ríos mediterráneos, con un fuerte estiaje durante los meses de julio $y$ agosto. Por consiguiente casi todas las zonas situad as por debajo o a nivel del lecho del río son susceptibles de inundación, y se secan durante los meses de verano hasta mediados de otoño. En la zona se encuentran una serie de cubetas excavadas hasta diferentes profundidades que se van secando progresivamente a lo largo de la estación seca a excepción de una que es permanente a lo largo del año. Todas las charcas están densamente pobladas por macrófitos con una gran diversidad de ambientes florísticos por las diferentes características de las aguas.

\section{MATERIAL Y METODOS}

El muestreo se realizó quincenalmente, desde setiembre de 1980 hasta octubre de 1981 , con la finalidad de seguir la evolución de las poblaciones de crustáceos a lo largo de su ciclo anual. La recogida de material se realizó con una red cónica de $28 \mathrm{~cm}$. de diámetro por $50 \mathrm{~cm}$. de longitud y de 250 micras de poro.

El muestreo se efectuaba haciendo pasadas

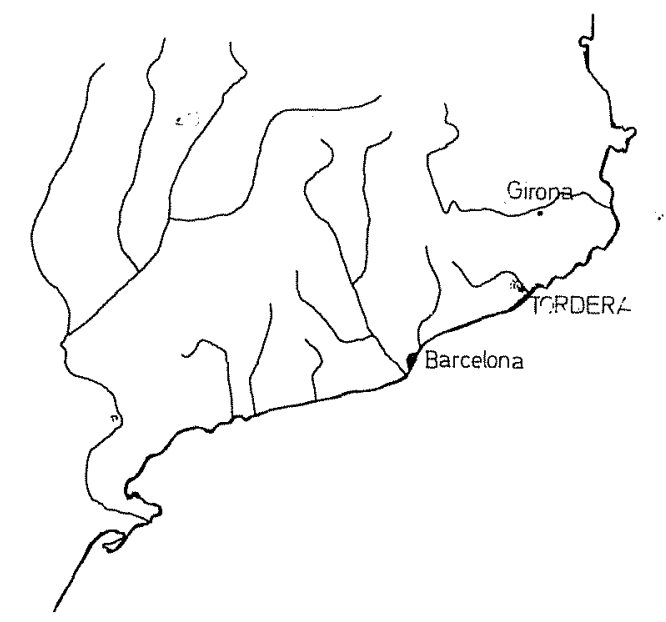

Fig. 1. Situación del área de muestreo.

en sentido horizontal, recorriendo en cada charca una distancia de $75 \mathrm{~cm}$. aproximadamente y procurando en cada ocasión tomar la muestra en el mismo lugar. Este criterio permite comparar las fluctuaciones de las densidades de las especies en las diferentes épocas del año, aunque no.permite una estima absoluta de dichas densidades.

El volumen filtrado por la red, es igual al producto de la sección de la boca de la red por la longitud recorrida, y por un factor de corrección o nivel de eficacia de un $40 \%$, puesto que para evitar la entrada de restos vegetales y hojas se interpuso una malla de mayor tamaño delante de la boca de la red cónica.

Las muestras recolectadas se fijaban con formaldehido al $4 \%$ y posteriormente, se identificaban las especies y se procedía a un recuento bajo un microscopio esteroscopio binocular. El recuento se hacía de una parte alícuota de la muestra no inferior a los 500 individuos.

Las áreas de muestreo escogidas están en relación al tiempo que tarda en secarse cada una de las zonas. Aunque existe una gran diversidad de ambientes, se han diferenciado 4 zonas bien distintas tanto en sus características hidrológicas, físico-químicas, como 
morfológicas que son: La laguna, los canales, la zona de los helófitos, y los prados inundados, de las cuales sólo la primera posee agua de forma permanente.

\section{AGUAS PERMANENTES}

El carácter permanente de la laguna se debe a que su cota mínima está muy por debajo de la cota del lecho del río. Tiene forma circular y ocupa una superficie de unos $600 \mathrm{~m}^{2}$ aproximadamente con una profundidad que oscila entre $1,5 \mathrm{~m}$. y $50 \mathrm{~cm}$., correspondiendo, respectivamente, al periodo invernal y estival. (fig. 2)

En los márgenes no sumergidos de la laguna se encuentra una gran diversidad de especies de helófitos entre las que domina Schoenoplectus lacustris ssp glaucus, mientras que la zona litoral propiame nte inundada está colonizada por macrófitos de la asociación Potamogeton denso-nodosi.

En el centro de la laguna, durante el otoño y el invierno, se desarrollan grandes masas de Lemna minor que flotan por la superficie de las aguas tranquilas. A mediados de enero de 1981 se produjo un crecimiento exhuberante de algas filamentosas (Spirogyra sp, Zygnema poliosporum, Oedogonium sp.) (COMELLES Comunicación personal), que invadió toda la laguna.

Es en esta zona donde se ha encontrado el mayor número de crustáceos, ya que la permanencia de agua todo el año permite el desarrollo de comunidades más estables en comparación con los otros tipos de ambientes estudiados (fig. 3 ).

La composición faunística de esta zona varía poco con el tiempo y está dominada principalmente por las siguientes especies: Herpetocypris chevreuxi, Eucyclops serrulatus, Megacyclops viridis, Diacyclops bicuspidatus odessanus, Ceriodaphnia reticulata, Scapholeberis mucronata, Tretocephala ambigua, Alonella excisa, Simocephalus vetulus y Chydorus sphaericus. Otras sólo se encuentran en determinadas épocas del año, debido a su carácter más estenotermo como Canthocamptus staphylinus, Cyclops strenuus strenuus, más propias de invierno, y de Thermo-
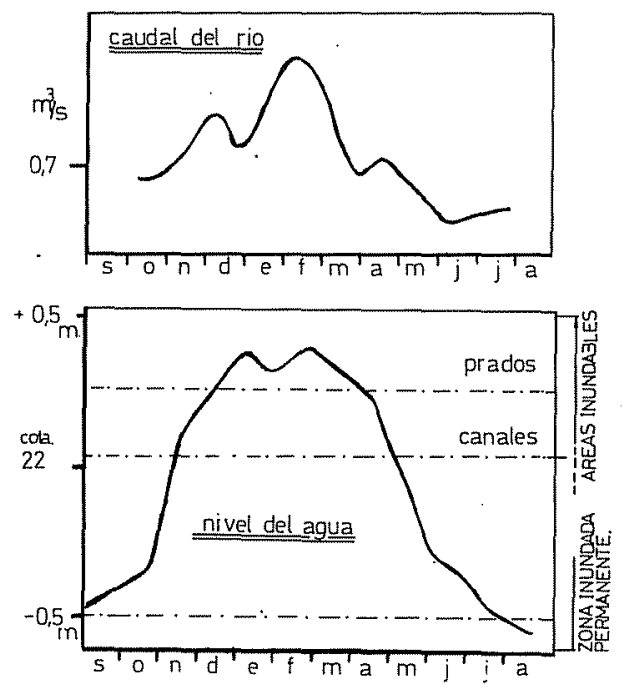

Fig. 2. Fluctuaciones del nivel del agua en la zona de "La Tordera".

cyclops dybowskii, Notodromas monacha y Macrocyclops fuscus en los períodos más cálidos.

Del conjunto de especies de esta zona, algunos crustáceos exigen cierta estabilidad hidrológica para poder desarrollar su actividad, y éstos son los que caracterizan mejor a la comunidad especialmente Daphnia longispina, Thermocyclops dybowskii, Macrocyclops fuscus, Eucyclops serrulatus, Herpetocypris chevreuxi, Notodromas monocha, $\mathrm{Mi}$ crocyclops varicans, Ceriodaphnia reticulata, que sólo se han encontrado en esta zona de aguas permanentes, si bien algunos han aparecido ocasionalmente en otros puntos de aguas temporales, en el momento en que toda la zona está inundada y es posible su difusión.

\section{AGUAS TEMPORALES}

Corresponden a las zonas estrictamente inundables ya que se hallan a una cota que es igual o inferior a la del nivel del lecho del río. El nivel del agua de estas zonas están íntimamente relacionad as con el caudal que lleva el río en cada momento, y se inundan cuando 


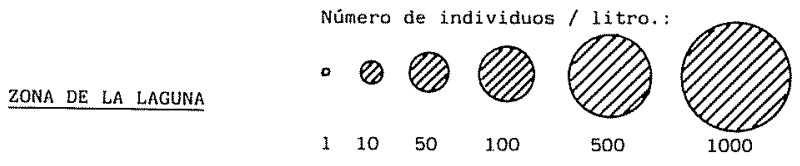

Ceriodaphnia reticulata. (Jurine, 1820).

$000: 00$.

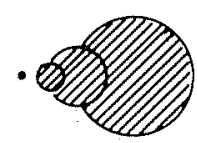

Daphnia langispina. (0.F. Muller, 1785).

Daphnia obtusa . (kurz,1874).

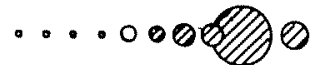

- Q0Q0

Scapholeberis kingi. (G.0.Sars, 1903).

Simocephalus vetulus. (O.F.Müller, 1776

Alonetla exeisa. (Fischer, 1854).

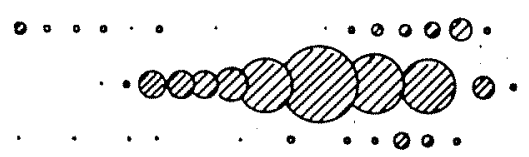

Chydorus sphaericus. (O.F.Mill 1er.1785).

Tretocephata ambigua. (Frey, 1965).

Mixodiaptomus kupelwieseri. (Brehm, 1907).

Macrocyclops fuscus. (Jurine,1820).

Eucyclops semmlatus. (Fischer, 1851).

Cyclops strenuus strenuus. (Fiscier, 1851).

Megacyalops viridis $v$. (Jurine, 1820)

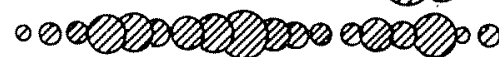

- - 000000000000000

Diacyclops bicuspidatus odessarus. (s

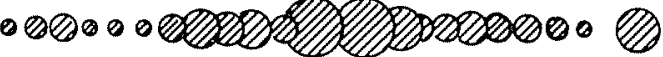

Thermocyetops dybouskii. (Landé, 1890).

Microcyclops waricans. (Sars, 1863).

Canthocamptus staphylinus. (Jurine, 1820)

Notodromas monocha. (0.F.Müil ler, 1.776).

-. 00000000000 .

llerpetocypris chevreuxi. (6.0.Sars, 1896).

$\oslash 00$

$000 . \quad 0$

Aseltus coxalis banyulensis. (Racovitza, 1919)

000

00

00.

. . . . . . 0000

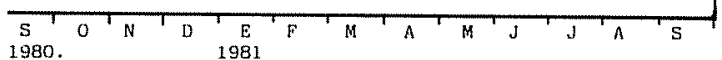

Fig. 3. Crustáceos presentes en la zona de la laguna y variación de su densidad a lo largo del año. 
Núnero de individuos/li tro.:

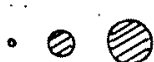

$110 \quad 50$

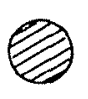

100

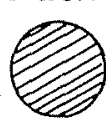

500

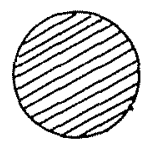

000

Ceriodaphnia retiaulata. (Jurine, 1820). Ceriodaphnia Laticaudata. (D.E.Miller, 1867).

Scapholeberis kingi. ( G.0. Sars,1903).

Alonella excisa. (Fischer, 1854)

Simocephalus vetulus. (0.F.Muller, 1776).

Chydorus sphaericus. (O.F.Muller,1785).

Pleuroxus laevis. (Sars,1862).

Tretocephala ambigua. (Frey, 1965).

Mixodiaptomus kupelwieseri. (Brehm,1907).

Megacyclops viridis v. (Jurine, 1820).

Diacyelops bicuspidatus odessanus. (Schankevitch,1875)

Canthocamptus staphylinus. (Jurine, 1820).

Cypris bispinosa. (Lucas, 1846).

E'ucypris virens. (Jurine, 1820).

Heterocypris incongruens. (Ramdohr, 1808).

Cypridopsis parva. (G.W.Muiller, 1900).

Asellus coxalis banyulensis. (Racovitza,1919).

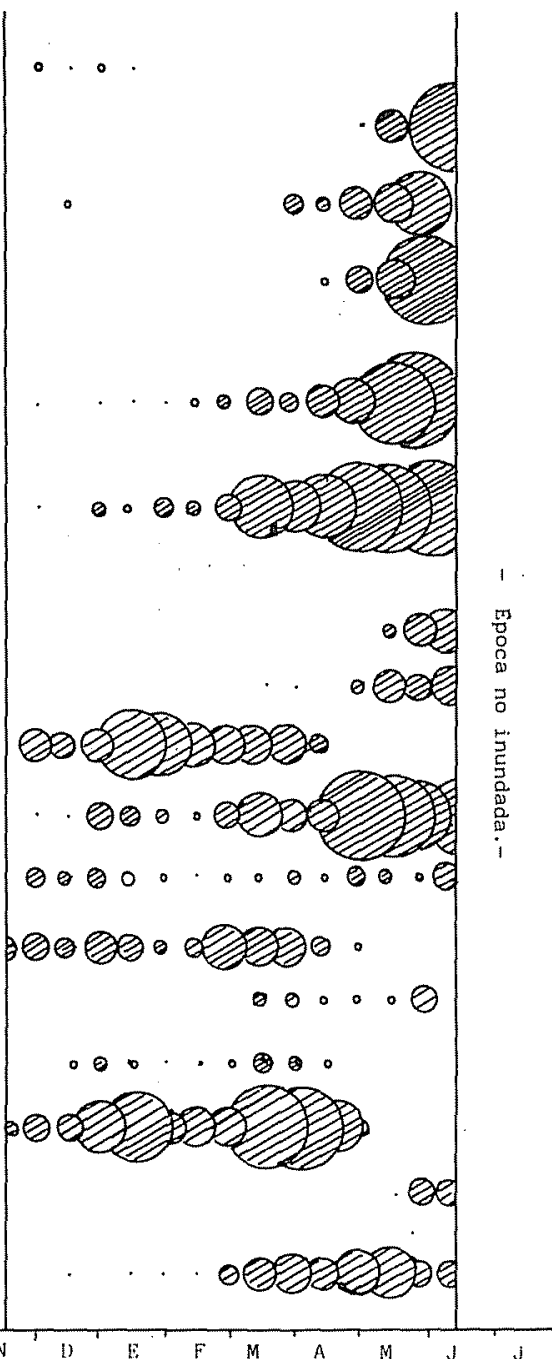

Fig. 4. Especies de crustáceos propios de la zona de canales y densidades que alcanzan en diferentes épocas del año. 
el río lleva un caudal igual o superior a $0,7 \mathrm{~m}^{3} / \mathrm{s}$ (fig. 2).

\section{CANALES}

Toda la zona está surcada por una red de canales que fueron construidos antiguamente con el fin de drenar los campos de cultivo que quedaban inundados. El agua circula por ellos muy lentamente, y desemboca en un tramo inferior del río.

Las dimensiones de estos canales son de unos $80 \mathrm{~cm}$. de profundidad media y $1,5 \mathrm{~m}$. de ancho. Desde principios de noviembre hasta mediados de junio quedan completamente inundados propiciando el desarrollo de algas filamentosas (Spirogyra sp, Oedogonium sp, Zygnema poliosporum) (COMELLES. Comunicación personal,) que se comportan como especies pioneras en la colonización de este tipo de ambientes llegando a invadir masivamente todos los canales a comienzos de primavera. Después de esta etapa, y ya en primavera, las algas son sustituidas por macrófitos que cubren completamente la superficie del agua. En los canales domina especialmente Callitriche polymorpha acompañada de Ranunculus fluitans, $G a$ lium palustre, Glyceria fluitans, mientras que en los márgenes se encuentran: Iris pseudacorus, Galium palustre, Equisetum arvense, Carex riparia, Scirpüs lacustris. (BARÓN, Comunicación personal).

La composición de crustáceos en estos ambientes varía bastante durante el año diferenciándose las especies pioneras en la colocazión (Heterocypris chevreuxi, Mixodiaptomus kupelwieseri) de otras más estables, que aparecen a continuación y durante la desecación de los canales a principios de verano. (fig. 4).

\section{ZONAS DE VEGETACION HELOFITA}

En toda la zona, la vegetación helófita es la que más extensión ocupa, distribuyéndose en enclaves monoespecíficos de gran expansión y formando un mosaico de comunidades muy variado. Una gran extensión está ocupada única y exclusivamente por Phragmites comunis, y por Schoenoplectus lacustris ssp glaucus acompañad de Carex riparia. En todas estas zonas la vegetación forma densos y altos cañizares que quedan inundados desde noviembre hasta junio, destacando una mayor profundidad de agua en las zonas ocupadas por Schoenoplectus lacustris ssp glaucus.

En zonas con poblaciones de helófitos, $\tan$ espesas, es muy difícil el desarrollo del fitoplancton por lo que los crustáceos que las habitan son principalmente bentónicos y detritívoros (fig. 5).

\section{PRADOS INUNDADOS}

Comprende a la zona situada a un nivel superior (fig. 2). Si bien se inunda periódicamente, la duración del agua encharcada es más corta que en los casos anteriores con un máximo de $25 \mathrm{~cm}$. de profundidad.

El tipo de vegetación es la característica de prados húmedos, con Juncus effusus, Eleocharis palustris y Trifolium pratense como especies dominantes (BARON, Comunicación personal).

Se trata, pues, de un sistema fluctuante muy poco estable, en el que la comunidad de crustáceos está muy poco evolucionada y fomada por las especies más euriocas (fig. 6).

Las especies de las tres zonas con aguas temporales que hemos delimitado que mejor caracterizan la comunidad de crustáceos son el diaptómido Mixodiaptomus kupelwieseri y los ostrácodos Eucypris virens, Heterocypris incongruens, Cypris bispinosa, Candona candida, Cyclocypris ovum y Cypridopsis parva.

Inicialmente este tipo de aguas son colonizadas por una serie de especies pioneras que se desarrollan con gran rapidez para ser, a continuación, desplazadas por otras especies, cuando las condiciones se estabilizan. Entre las especies del primer grupo destacan: Heterocypris incongruens, Candona candida, Mixodiaptomus kupelwieseri, Canthocamptus staphylinus y Cyclops strenuus strenuus, y entre las que las sustituyen se encuentran:

$$
\because *
$$


Nümero de individuos/litro:
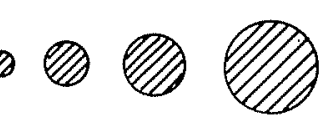

$1 \quad 10 \quad 50 \quad 100$

$$
500
$$

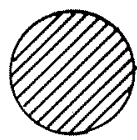

Ceriodaphnia laticaudata. (D. E.Muller, 1867)

Ceriodaphnia reticulata. (Jurine,1820).

Daphnia obtusa. (Kurz,1874).

Stapholederir. kingi. (G.0.Sars, 1903).

simocephalus vetılus.(O.F.Muller, 1776)

Chydorus sphaericus. (0.F.Muller, 1776).

Mixodiaptomus kupelwieseri. (Brehm, 1907).

Cyctops strenuus strenuus. (Fischer, 1851).

Meguelyclops viridis 1$)$ (Jurine,1820).

Diacyelops bicuspidatus odessanus. (Schankevitch,1875).

canthocamptus staphylinus. (Jurine,1820).

Candona candida. (G.W.Mill ler, 1900).

(yelocypris ovum. (Jurine, 1920).

lleterocypris incongruens. (Ramdohr, 1808).

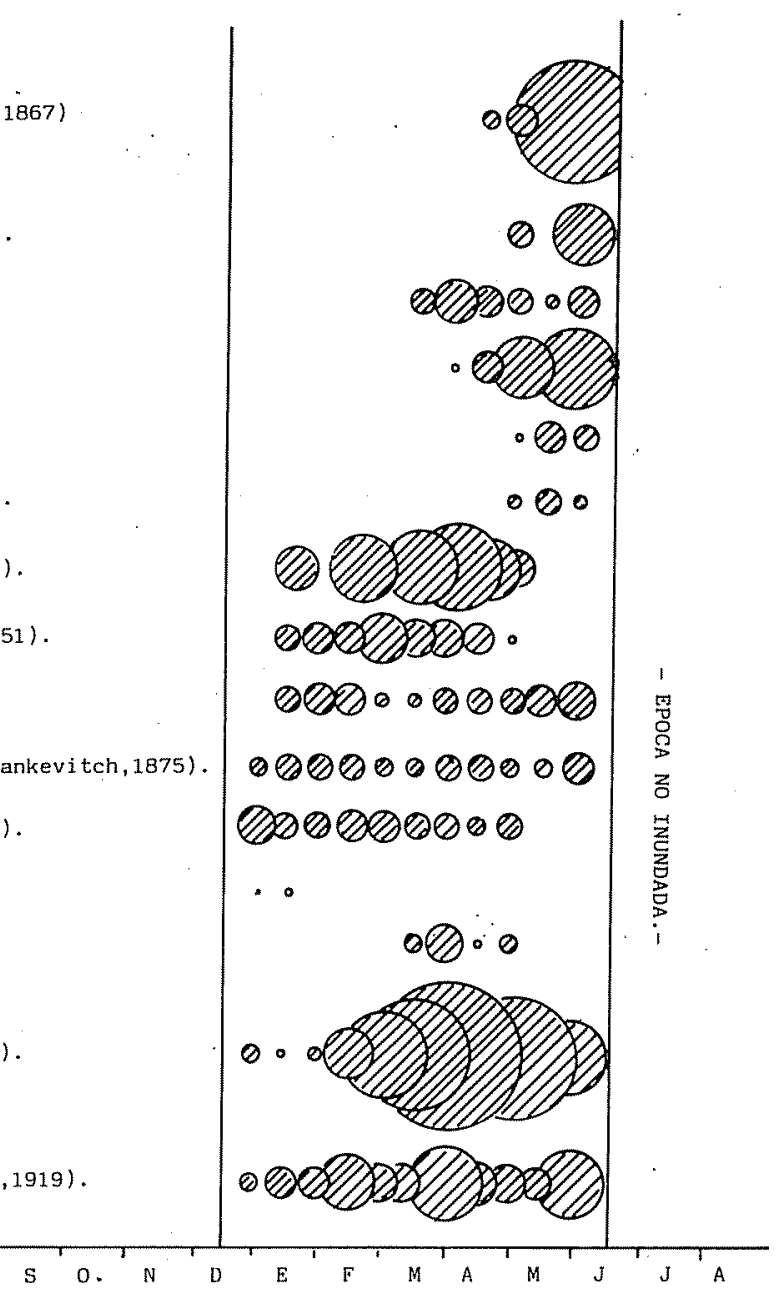

Atiellus coraliz; banyulensis. (Racovitza,1919).

Fig. 5. Comunidades de crustáceos de la zona de helófitos durante el tiempo que permanecen inundados. 

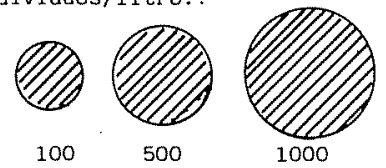

Scapholeberis kingi. ( G.0.Sars, 1903).

Simocephalus vetulus. ( O.F.Miller,1776).

Alonella excisa (Fischer, 1854).

Chydorus sphaericus. (0.F. Muller, 1785).

Tretocephala ambigua. (Frey, 1965).

Mixodiaptomus kupetwieseri. (Brehm, 1907).

Eucyclops serrulatus. (Fischer,1851).

Megaeyclops viridis v. (Jurine, 1820).

Diacyclops bicuspidatus odessanus. (Schankevitch,1875).

Candona candida. (G.W.Miiller, 1900).

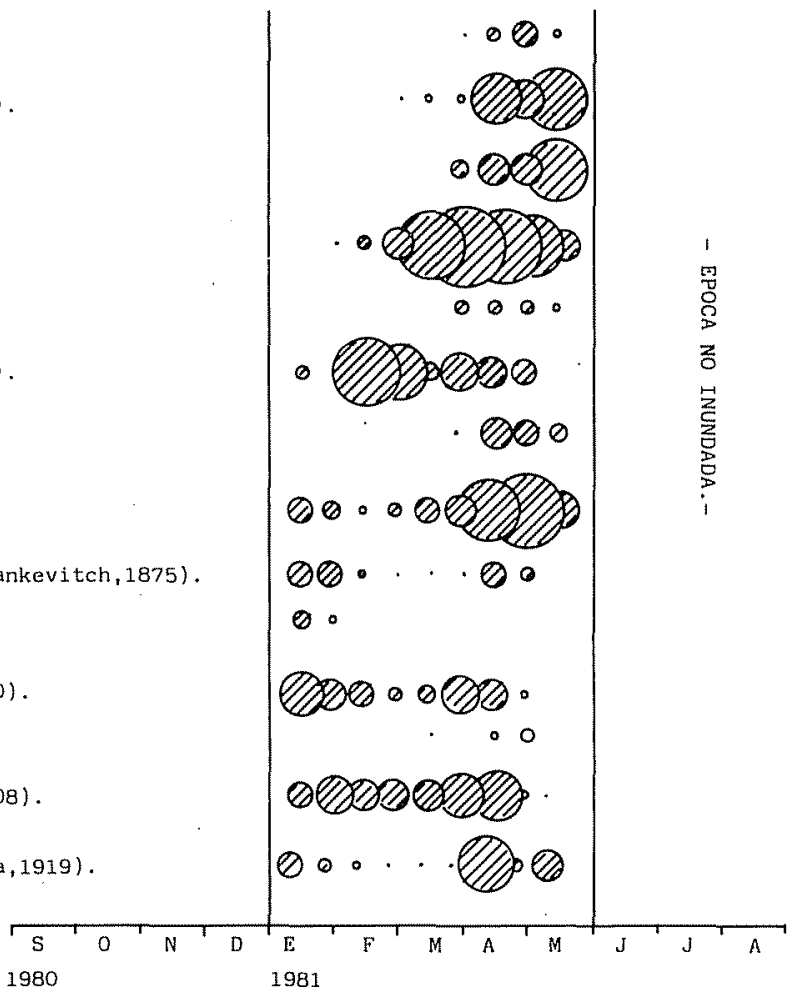

Canthocamptus staphylinus. (Jurine,1820).

Cypris bispinosa. (Lucas, 1846).

Heterocypris incongruens, ( Ramdohr, 1808).

Asellus coxalis banyulensis.( Racovitza,1919).

Fig. 6. Crustáceos de los prados que se inundan en la zona de "La Tordera" y densidades en las que se presentan a lo largo del año.

Cypridopsis parva, Cypris bispinosa, Asellus coxalis banyulensis, Megacyclops viridis y Diacyclops bicuspidatus odessanus (figs. 4 a 6). También cabe destacar que Ceriodaphnia laticaudata y Pleuroxus laevis sólo han sido localizad as en aguas temporales.

La composición química global del agua es muy uniforme en toda la zona, en especial respecto a la mineralización (Sulfatos, clorulos, alcalinidad) si bien hay pequeñas dife- rencias en el comportamiento de estos parámetros que es conveniente destacar. La mineralización aumenta a medida que las charcas se secan. Como la duración del agua en cada una de las zonas es distinta, unas se mineralizan más rápidamente que las otras. Otro factor importante es la circulación del agua ya que si ésta circula rápidamente facilita la eliminación de las sales acumuladas. Esto explicaría que en la laguna, con una 
tasa de renovación menor la concentración de sales a finales de verano sea muy grande en comparación con el resto (fig. 7).

Otros parámetros físico-químicos, el $\mathrm{pH}$; la temperatura; la concentración de fosfato, de nitritos, de nitratos y el oxígeno disuelto, registran fluctuaciones diarias muy diferentes en cada una de las tres áreas muestreadas. En dos experiencias realizadas, una en febrero y otra en marzo, en la que se recogían muestras cada tres o cuatro horas durante todo el día, se constató que las variaciones de cada uno de los parámetros eran más bruscas en el prado inundado y más amortiguadas en la laguna. Ello es comprensible ya que en las zonas donde hay poca profundidad y existen sucesivas variaciones de nivel de agua (como ocurre en los prados inundados) no puede desarrollarse un ambiente con cierta estabilidad y por tanto se producen grandes fluctuaciones ambientales.

\section{DISCUSION}

La distribución en especies de crustáceos en relación a las características hidrológicas -aguas permanentes, aguas temporales, duración media de las inundaciones, y proximidad de la capa freática- es un sistema útil para tipificar cada uno de los ambientes estudiados. Así por ejemplo, si un nivel bajo en inundación es un elemento desfavorable para el desarrollo de los crustáceos planctónicos, no lo es en cambio, para los propios del bentos. Por otra parte el desarrollo de una comunidad más estable precisa de condiciones hidrológicas más constantes.

La mayoría de las especies de crustáceos identificadas en "La Tordera" han sido también citadas por diversos autores (MARGALEF 1953,PROSZYNSKA 1962b,DUSSART 1969, FLÖSSNER 1972, BRONSTEIN 1947, TETART 1974, ARMENGOL 1978) en aguas someras $y$ en zonas muy ricas en vege tación acuática $y$ en general se trata de especies eurioicas.

El tipo de comunidad que se encuentra en la laguna presenta características más estables y no es tan susceptible a las fluctuaciones del

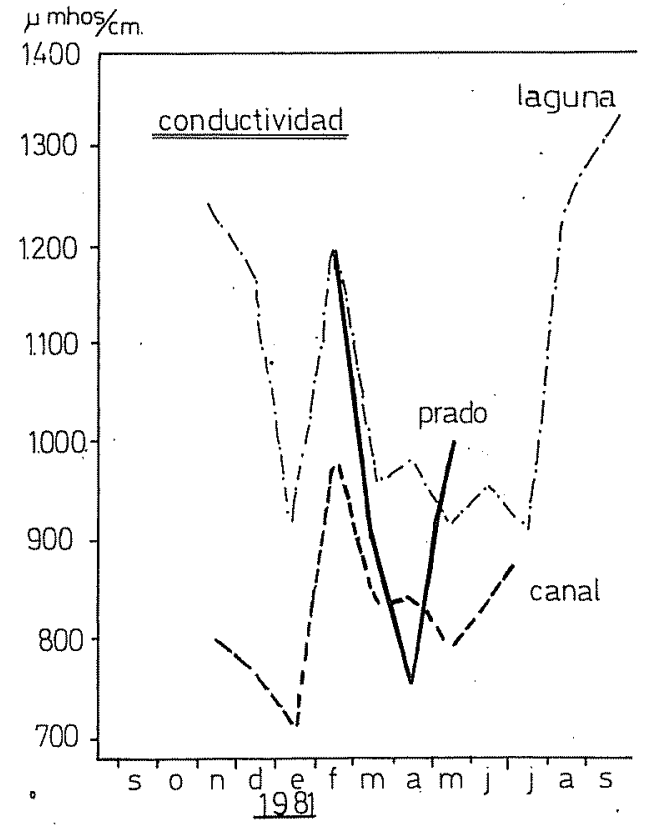

Fig. 7. Mineralización del agua en los diferentes medios estudiados.

nivel del agua. Se trata de especies que, en general, han sido descritas en zonas litorales de los lagos y lagunas, entre la vege tación sumergida y emergida. Quizás la especie con más preferencia a una vida típicamente planctónica sea Daphnia longispina, aunque ha sido considerada por varios autores como eurioica (MARGALEF 1953, ARMENGOL 1978, PACAUD 1939, PROSZYNSKA 1962a). Thermocyclops dybowskii es otra especie de comportamiento similar a la anterior, ya que ha sido citada tanto en el plancton como en la zona litoral de lagos (DUSSART 1969, GURNEY 1933). Otras especies como Eucyclops serrulatus y Macrocyclops fuscus se encuentran en la laguna por su incapacidad de resistir la desecación, si bien la primera parece sobrevivir en estado adulto, la falta de agua por espacio de un mes siempre y cuando el sedimento conserve cierta humedad (MARGALEF 1953. SPANDL 1925).

En contraposición a las especies exclusi- 
vas de las aguas permanentes existen aquéllas que han podido desarrollar adaptaciones a los medios fluctuantes, como las aguas temporales, ya sea adoptando formas de resistencia a la sequía, o bien ajustando la duración de su vida activa al periodo de inundación. Suelen ser especies univoltinas y de desarrollo monocíclico. En los prados inundados, donde las condiciones de sequía son bastante severas, el grupo de crustáce os que se desarrollan después de la inundación, son aquéllos que superan el período de sequía mediante huevos de larga duración. Este es el caso de los cladóceros, de algunos ostrácodos como Candona candida, Eucypris virens y Heterocypris incongruens y del diaptómido Mixodiaptomus kupelwieseri. Los canales y en las zonas con helófitos, donde el periodo de sequia no es tan drástico puesto que la capa freática se aleja poco de la superficie del suelo, permiten el desarrollo de aquellas especies que superan este período en estado de latencia enterrados en el fango como Cyclops strenuus strenuus, Canthocamptus staphylinus, Diacyclops bicuspidatus odessanus, Megacyclops viridis y Cyclocypris ovum (WIERZBICKA 1962-66, STELLA \& MARGARITORA 1968, CHAMPEAU 1970, EL GMORK 1955). Cyclops strenuus strenuus aparece inmediatamente después de la inundación en estadios de copepodito IV-V con un ciclo univoltino y de esta manera se adapta al carácter temporal de las inundaciones. Esta particularidad le da una posición privilegiada ya que siendo carnivora le permite, durante esta primera época, explotar las poblaciones nauplianas o inmaduras de otros crustáceos que están presentes en la fase inicial de las inundaciones (PROSZYNSKA 1962a, TETART 1974). Para Canthocamtus staphylinus se ha dicho que pasa el período de sequía estival en estado adulto, siendo los individuos femeninos fecundados los que soportan este período'(DONNER 1928,SMYL 1957). Lo mismo se ha descrito para Cyclops strenuus strenuus (ELGMORK 1955). También son los adultos de Cyclocypris ovum los que sobreviven enterrados entre los sedimentos húmedos durante el período seco. Esto explica en parte su ausencia en aquellas zonas con período largo 'de sequía (TETART 1974).

Es de resaltar la gran proporción de ostrácodos que se encuentran en estas zonas inundables en comparación con las otras más permanentes. Esto se debe en parte a que estas especies son de desarrollo rápido y sus huevos son desecables por lo que pueden reconstruir sus poblaciones con rapidez. Su régimenen bentónico y detritívoro también favorece su mayor adaptacion. Eucypris virens incluso parece que sea capaz de ingerir minerales que se encuentran en el sedimento (TETART 1974).

La mayorfa de las especies encontradas en esta zona son cosmopolitas, pero existen dos que tienen un interés biogeográfico particular ya que su área de distribución dentro de la península Ibérica se limita por el momento a la provincia de Girona, en las zonas más cercanas al litoral costero. Una de ellas es la Tretocephala ambigua que resulta ser una nueva cita para la península Ibérica, $y$ la otra es Mixodiaptomus kupelwieseri que es considerada como especie característica de aguas temporales (DUSSART \& AGUESSE, 1956) y poco mineralizadas.

\section{BIBLIOGRAFIA}

AGUESSE, P. 1956. Quelques considérations sur les cópedodes de la Camargue. Vie et Milieu, VII (1): 38-42.

ALONSO, M. \& ARMENGOL , J. 1981. Distribució dels diaptómids ibèrics en relació amb àrees geogràfiques próximes: Factors històrics i ecològics. Treb. Inst. Cat. Hist. Nat., 9: 135-145.
ARMENGOL J 1972. Ostràcods de la vall de Bigues. Treb. Soc. Cat. Biol, XXXII: 121-125. 1977. Los crustáceos de las aguas del valle de Bigues (Barcelona). Graellsia., XXXI: 235246.

1978. Los crustáceos del plancton de los embalses españoles. Oecologia aquatica, 3: 3-96. 
BRONSTEIN, Z.S. 1947. Ostracodes des eaux douces. Faune de l'URSS. II. Academ. Sciences URSS. Moscou. 340 págs.'

CHAMPEAU, A. 1970. Recherches sur l'écologie et l'adaptation à la vie latente des copépodes des eaux temporaires provençales et corses, "Thèse, Université d'Aix-Marseille."

DONNER, F. 1928. Die Harpactizeden der leipziger umgehundg und der schneeberger erzbergwerche. Int. Rev. Hydrobiol., 20: 221-353.

DUSSART, B. \& AGUESSE, P. 1956. Sur quelques crustaces de Camargue et leur écologie. Vie et Milieu, VII : 481-520.

DUSSART, B. 1967. Les copépodes des eaux continentales d'Europe Occidentale. Vol. I Boubée. París. 500 págs.

1969. Les copépodes des eaux continentales d'Europe Occidentale. Vol. II Boubée. París. 292 págs.

ELGMORK, K. 1955. A resting stage without encystment in the annual cycle of the freshwater copepod Cyclops strenuus strenutus. Ecology Vol. 36. 4: 739-743.

FLOSSNER, D. 1964. Zur cladocerenfauna des Stechlinsee-Gebietes. II. Ökologische Üntersuchungen über die litoralen Arten. Limnologica. 2: 35-103

1972. Krebstiëre (Crustacea). Kiemen-und Blattfüsser, Branchipoda, Fischläuse, Branchiura: Die tiernvelt Deustchlands, 60:1-501.

GURNEY, 1933. British freshwater copepoda. III "Cylopoida". The Ray Society. London, 384 "CP.

MARGALEF, R. 1950. Algunos crustáceos interesantes de las aguas dulces y salobres de España. Publ. Inst. Biol. Apl., 7: 131-152.

1953. Los cristáceos de las aguas continentales ibéricas. Biología de las aguas continentales, 10. Minist. Agric. Inst. Forest. Invest. y Exp. Madrid, 243 págs.

1974. Ecología. Omega, Barcelona, 951 págs.

NEGREA, S. \& NEGREA, A. 1975. Ecologia Po pulatililor de Cladoceri si gasteropode din zona inundabilá a Dunárii. Ed. Academ. Republici Socialiste România. Bucvresti. 232 págs.

PACAUD, A. 1939. Contribution à l'écologie des cladocéres. Bull. Biol. Fr. Belg. Suppl., 25: 1260.

PETIT \& SCHACHTER. 1954. La Camargue, étude écologique et faunistique. Ann. Biol., 30 (5-6): 193-253.

PROSZYNSKA, M. 1962a. Cladocers and copepods of water bodies of Tatra montains and Podhale Region some remarks on typology of pools. Int of Zoology of Polish Academy of Science. Warsowa. 1. VIII.: 157-166.

1962b. The annual cycle in occurrence of Cladocera and Copepoda in Small Water Bodies. Polsk. Arch. Hydrobiol. X (XXIII): 379-422.

SCOURDFIELD, DJ, \& HARDING, JP, 1958. A key to the British species of freshwater Cladocera with notes on their ecology. (2nd. ed.) Freshwater Biol. Ass. Sci. Publ., 5: 55 págs.

SMIRNOV, N.N. 1971. Chydoridae of the world's. Fauna de la URSS. serie no 101.529 págs.

SMYLY, W.J.P. 1957. Distribution and seasonal abundance of entomostraca in moorland ponds near windermere. Publ. Freshw. Biol. Ass. 11: 50-72.

SPANDL, H. 1926. Copepoda and branchiura. Bio1. Tiere Deutschlands $15.82 \mathrm{pp}$.

STELLA, E. \& MARGARITORA, F. 1968. La fauna ad entomostraci di acque astatiche del Lazio Acad. Nazionale dei XL serie IV. Vol. XVIII: 89.

TETART, M. 1974. Les Entomostracés des milieux peu profonds de la vallée du Rhône. Essai d'étude écologique: compositions des associations et répartition des espèces. Trav. Lab. Hydrob. Pisc. Grenoble, 64-65: 109-245.

WIERZBICKA, M. 1962. On the resting ștage and mode of life of some species of "Cyclopoida". Pol Arch. Hydrobiol. 10: 215-229.

- -1966. Les résultats des recherches concernant l'état de repos (resting-stage) des Cyclopoida. 4 Verh. Internat. Verein Limnol., 16: 592-599. 